\title{
Clinical Role of Non-Contrast Magnetic Resonance Angiography for Evaluation of Renal Artery Stenosis
}

\author{
Daisuke Utsunomiya, MD; Mitsue Miyazaki, PhD*; Yohei Nomitsu, RT; Yosuke Komeda, RT; \\ Takashi Okigawa, RT; Joji Urata, MD; Yasuyuki Yamashita, MD**
}

\begin{abstract}
Background The association between a gadolinium-based contrast material and nephrogenic systemic fibrosis has been discussed. The purpose of our study was to evaluate whether non-contrast enhanced magnetic resonance angiography (MRA) might provide sufficient information of renal artery stenosis.

Methods and Results The non-contrast MRA of 26 patients with hypertension was retrospectively reviewed in the present study. The significant renal artery stenosis was visually evaluated by comparing non-contrast MRA with computed tomography or conventional angiographic finding. Difference of the intensities between the proximal and distal aorta was quantitatively evaluated. The sensitivity, specificity, positive predictive value and negative predictive value of non-contrast MRA in the evaluation of the renal artery stenosis was 78\%, 91\%, 64\% and 96\%, respectively. The distal abdominal aorta showed less signal intensity than the proximal aorta by $16.9 \pm 12.2 \%$.

Conclusions Non-contrast MRA is a non-invasive and effective method that allows evaluation of the renal artery stenosis. (Circ J 2008; 72: 1627-1630)
\end{abstract}

Key Words: Angiography; Hypertension; Magnetic resonance imaging; Renal

$\mathbf{R}$ enal artery stenosis is a potentially curable cause of hypertension and impaired renal function. Although conventional angiography remains the reference standard for the diagnosis of renal artery stenosis, it is invasive and not used as a screening examination. Of the less invasive alternatives, contrast-enhanced computed tomographic angiography (CTA) and magnetic resonance angiography (MRA) have the best accuracy in diagnosis of renal artery stenosis $!^{1-3}$ However, CTA has the distinct disadvantages of ionizing radiation and nephrotoxic contrast material.,5 Recently, the association between a gadolinium-based contrast material and nephrogenic systemic fibrosis (NSF) has been discussed 6,7 The clinical course of NSF can be progressive and might be fatal? Therefore, non-contrast MRA has increasingly become important in clinical settings. Noncontrast MRA using 3-dimensional steady-state free precession (SSFP) has the potential to be an alternative screening method for renal artery stenosis. The purpose of our study was to evaluate whether non-contrast enhanced MRA might provide sufficient information of renal artery stenosis.

\section{Methods}

Patients

The records in our radiology database from the period

(Received January 9, 2008; revised manuscript received April 28, 2008; accepted May 15, 2008; released online August 27, 2008)

Diagnostic Imaging Center, Saiseikai Kumamoto Hospital, Kumamoto, Japan, *Department of Advanced Clinical Research, MR, Toshiba Medical Research Institute, Vernon Hills, IL, USA and **Department of Diagnostic Radiology, Graduate School of Medical Sciences, Kumamoto University, Kumamoto, Japan

Mailing address: Daisuke Utsunomiya, MD, Diagnostic Imaging Center, Saiseikai Kumamoto Hospital, 5-3-1 Chikami, Kumamoto 8614193, Japan. E-mail: daisuke-utsunomiya@ saiseikaikumamoto.jp All rights are reserved to the Japanese Circulation Society. For permissions, please e-mail: cj@j-circ.or.jp between June 2006 and July 2007 included MRIs of 26 patients (17 men and 9 women; mean age, 61 years; age range, 42-79 years) who underwent non-contrast renal artery MRA. Twenty-four of these patients underwent renal artery CTA and 2 patients underwent conventional angiography. The main complaint of these patients was hypertension. All patients were referred to our department for magnetic resonance imaging (MRI) to evaluate the renal arteries. The retrospective review study was approved by the institutional review board.

MRI Technique

MRI was performed with a $1.5-\mathrm{T}$ superconductive unit with $33 \mathrm{mT} / \mathrm{m}$ amplitude, $200 \mathrm{mTm} \cdot \mathrm{m}^{-1} \cdot \mathrm{ms}^{-1}$ slew rate (Excelart Vantage XGV, Toshiba, Tokyo, Japan). The renal

Table 1 Imaging Parameters for Non-Contrast MRA With Time-SLIP SSFP

\begin{tabular}{lc}
\hline \hline Coil & Torso body \\
Orientation & Axial \\
Technique & $3 D$ true SSFP \\
No. of segment & 2 \\
Profile order & Interleave \\
Fat suppression & Chemical saturation \\
TR/TE $(\mathrm{ms})$ & $5.0 / 2.5$ \\
TI $(\mathrm{ms})$ & $1,300-1,500$ \\
Field of view $(\mathrm{mm})$ & $320 \times 320$ \\
No. of slices & 32 \\
Matrix & $256 \times 256$ \\
Bandwidth $($ Hz/pixel $)$ & 781 \\
SENSE factor & 2.0 \\
Scanning time $(\mathrm{s})$ & 192 \\
True acquired voxel size & $1.3 \times 1.3 \times 3.0$ \\
Reconsructed voxel size & $0.7 \times 0.7 \times 1.5$ \\
\hline
\end{tabular}

MRA, magnetic resonance angiography; SLIP, spatial spin labeling pulse; SSFP, steady-state free precession; TR, repetition time; TE, echo time; TI, inversion time; SENSE, sensitivity encoding parallel imaging. 


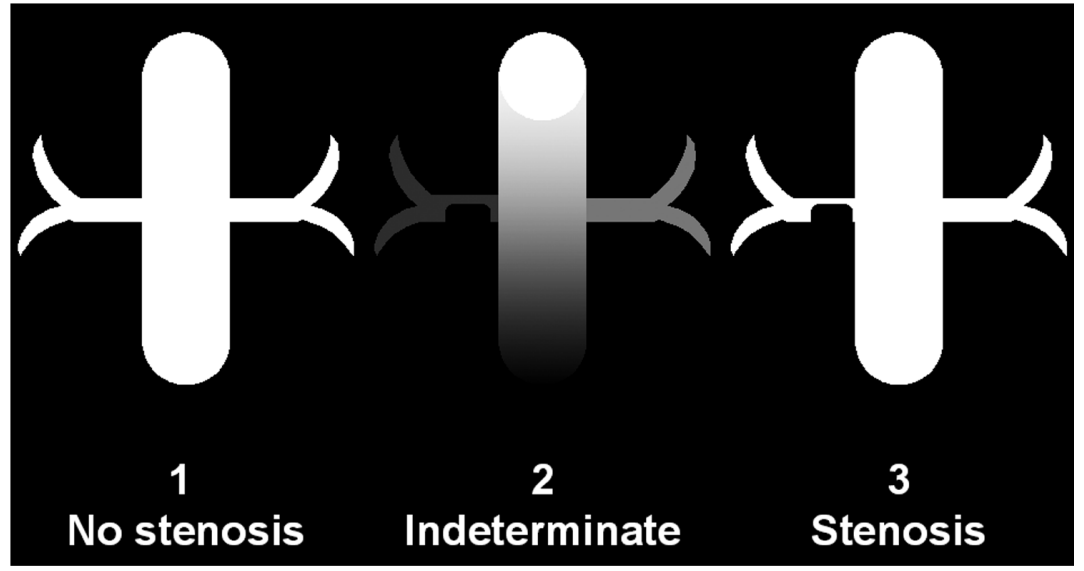

Fig 1. Schematic representation of visual evaluation scoring.
Table 2 Visual Evaluation Score for Non-Contrast MRA

\begin{tabular}{lcccc}
\hline \hline & \multicolumn{4}{c}{ Score } \\
\cline { 2 - 5 } & 1 & 2 & 3 & Undetectable \\
\hline Right $R A$ & 19 & 0 & 7 & 2 \\
Left $R A$ & 23 & 3 & 2 & 0 \\
Overall & 42 & 3 & 9 & 2 \\
\hline
\end{tabular}

Undetectable, accessory renal artery undetectable on non-contrast MRA.

$R A$, renal artery. Other abbreviation see in Table 1.

arteries were first localized with a SSFP coronal scout. After this, each patient underwent a free-breathing SSFP scan with respiratory gating. Relevant SSFP parameters are shown in Table 1. Free-breathing SSFP scanning was performed with time-spatial spin labeling pulse (time-SLIP) technique using a combination of a non-selective inversion pulse and a selective tag or marker pulse. A non-selective inversion pulse inverts all signals within a coil region to $-\mathrm{Mz}$ longitudinal magnetization. Immediately after the inversion pulse, a second spatially selective inversion pulse is applied that spins in the tagged region to bring back to the $+\mathrm{Mz}$ direction. In the result, the longitudinal magnetization in the tagged region is restored to $+\mathrm{Mz}$, whereas the magnetization elsewhere stays at $-\mathrm{Mz}$ and recovery back to $+\mathrm{Mz}$ as following the T1 recovery. After this tagging or marking period, a variable delay time (TI) is waited for the tagged blood to travel and then the data window is taken place using 3-dimensional balanced SSFP. An inferior saturation band pulse was applied to suppress inferior vena cava, renal vein and intestine signals. The travel time or TI was set around 1,300-1,500 ms for suppression of background signals to have an appropriate contrast between "renal parenchyma" is more preferable.

\section{Image Interpretation}

All MRA were retrospectively reviewed by 2 radiologists who did not know the results of CTA and conven- tional angiography which were considered as the reference standard. Neither reviewer was involved in the imaging interpretation of the cases before beginning this study. Reviewers visually evaluated the significant renal artery stenosis on the following 3-point criteria: 1, (no stenosis) indicated that the image quality was good with sufficiently high arterial intensity and there was no stenosis; 2, (indeterminate) indicated that the image quality was marginally acceptable with decreased arterial intensity and there was indeterminate stenosis; 3 , (stenosis) indicated that the image quality was good with sufficiently high arterial intensity and there was stenosis. Schematic representation of visual evaluation scoring is shown in Fig 1. The evaluations of the renal artery MRA were carried out by the consensus of 2 reviewers. We used a scan score of 3 to indicate significant stenosis and a score of 1-2 to indicate no significant stenosis in this analysis.

Imaging quality of non-contrast MRA with time-SLIP SSFP can be influenced by the in-flow phenomenon. Thus, difference of the intensities between the proximal and distal aorta was quantitatively evaluated. One radiologist performed operator-defined region of interest (ROI) measurements of the mean intensity of the proximal $(3 \mathrm{~cm}$ superior to the level of renal arteries) and distal aorta $(3 \mathrm{~cm}$ inferior to the level renal arteries) on non-contrast MRA. Circular ROIs were drawn to include the largest area in the ROI. The proximal-distal aorta signal intensity ratio (SIR) was defined as follows: $(\mathrm{Sp}-\mathrm{Sd}) / \mathrm{Sp} \times 100(\%)$, where $\mathrm{Sp}$ indicates signal intensity of the proximal aorta, and $\mathrm{Sd}$ indicates signal intensity of the distal aorta.

\section{Results}

Of the 26 patients, 9 had renal artery stenosis. All main renal arteries were detected on non-contrast MRA with time-SLIP SSFP. Four patients had accessory renal arteries. A total of 56 renal arteries (28 right renal arteries; 28 left renal arteries) were visually evaluated. Diagnostic

Table 3 Diagnostic Accuracy for Non-Contrast MRA

\begin{tabular}{lllll}
\hline \hline & Sensitivity & Specificity & PPV & NPV \\
\hline Right $R A$ & $5 / 5(100 \%)$ & $19 / 23(83 \%)$ & $5 / 9(56 \%)$ & $19 / 19(100 \%)$ \\
Left $R A$ & $2 / 4(50 \%)$ & $24 / 24(100 \%)$ & $2 / 2(100 \%)$ & $24 / 26(92 \%)$ \\
Overall & $7 / 9(78 \%)$ & $43 / 47(91 \%)$ & $7 / 11(64 \%)$ & $43 / 45(96 \%)$ \\
\hline
\end{tabular}

PPV, positive predictive value; NPV, negative predictive value. Other abbreviations see in Tables 1,2. 
accuracy in the detection of renal artery stenosis was evaluated for each artery. Results of visual evaluation scoring on MRA were presented in Table 2. The diagnostic accuracy results are presented in Table 3 . There were 4 false-positive cases and 2 false-negative cases. Two accessory renal arteries were undetectable on non-contrast MRA, and they were counted as false-positives. The visual evaluation scale of 2 false-negative cases was 2 (indeterminate). Representative cases are shown in Figs 2, 3 and 4.

On non-contrast MRA, the proximal-distal SIR was $16.9 \pm 12.2 \%$ (mean \pm SD). The distal abdominal aorta showed less signal intensity than the proximal aorta in 23 of 26 patients $(88 \%)$. Five patients had the proximal-distal aorta SIR of more than $25 \%$. The accessory renal arteries, which were undetectable on non-contrast MRA, arose from the distal abdominal aorta.

\section{Discussion}

Contrast-enhanced MRA has been evaluated more extensively than CTA for the assessment of renal artery stenosis, probably because many patients with renovascular disease have compromised renal function and therefore at higher risk for receiving iodinated contrast. However, the gadolinium-based contrast agent might trigger NSF in patients with renal impairment? Recently, non-contrast MRA with 3-dimensional SSFP technique has allowed for good visualization of renal arteries $!^{10}$ In the present study, non-contrast MRA with time-SLIP SSFP technique was evaluated.

Our study results showed that non-contrast MRA with time-SLIP SSFP technique showed high specificity (91\%) and negative predictive value $(96 \%)$ for the evaluation of renal artery stenosis. This indicates negative finding for renal artery stenosis on non-contrast MRA highly suggests true-negative. However, the lower positive predictive value $(64 \%)$ indicates non-contrast MRA tends to overestimate the renal artery stenosis. The previous report showed sensitivity and specificity on contrast-enhanced MRA was 93\% and $90 \%$, respectively! ${ }^{1}$ Our results with non-contrast MRA showed comparable specificity to contrast-enhanced MRA. We consider that non-contrast MRA might have a potential role as a screening technique for renal artery stenosis. Furthermore, the method is easy and fast to use, and it permits repeated imaging. However, the tendency of decrease of signal intensity in the distal aortic region was observed on non-contrast MRA with time-SLIP SSFP. This might be mainly because of the in-flow phenomenon and cardiac phase. Further technical improvement such as addition of cardiac gating should be necessary to increase the sensitivity, although sensitivity of $78 \%$ might be acceptable.

Depiction of accessory renal arteries is a challenge for MRA. In our study, 2 out of 4 accessory renal arteries were missed on non-contrast MRA. These 2 arteries are very small and arose from the distal abdominal aorta. Such small arteries can be possibly missed. Combined assessment with an additional breath-hold SSFP scan might be preferable. Furthermore, in order to confirm the presence of accessory renal artery, CTA with multidetector CT can be helpful because the high temporal and spatial resolution of the CT scanner with 16 or more detectors allows excellent angiographic images by a low-dose contrast material. ${ }^{12}$

There are several limitations in our study. First, our study group was small and the study was performed in a single institution on a single MR scanner. The multi-institution prospective study with larger study population should be

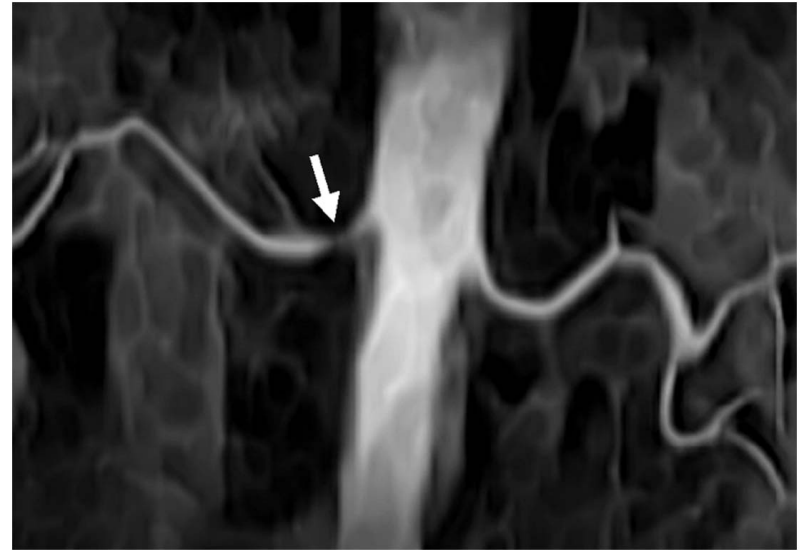

Fig 2. Non-contrast magnetic resonance angiogram of a 74-year-old woman with hypertension shows proximal renal arterial stenosis (arrow) (score 3).

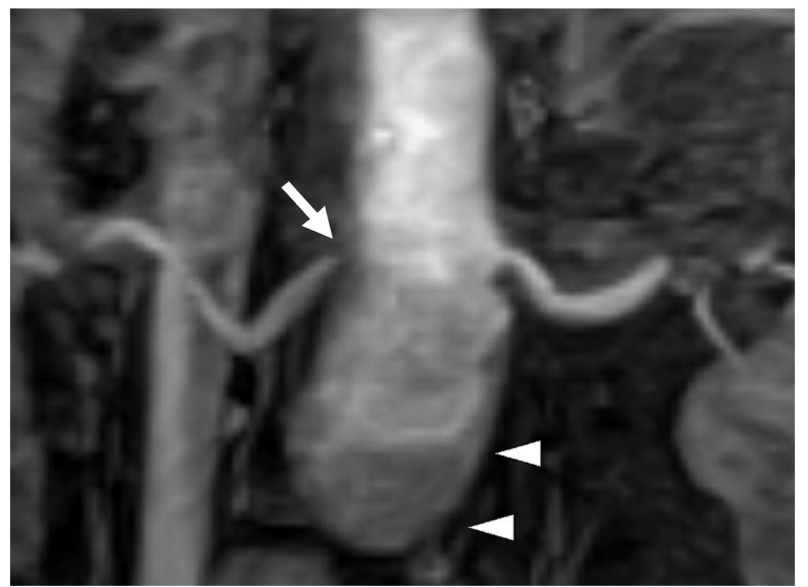

Fig 3. Non-contrast magnetic resonance angiogram of a 61-year-old man with hypertension and abdominal aortic aneurysm shows indeterminate renal arterial stenosis (arrow) (score 2). The distal abdominal aorta shows decreased signal intensity than the proximal aorta (arrowheads).

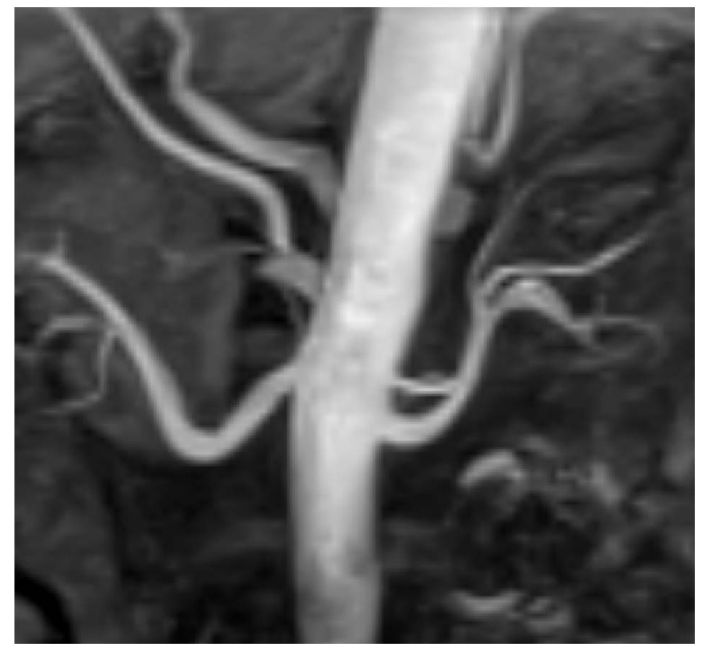

Fig 4. Non-contrast magnetic resonance angiogram of a 43-year-old woman with suspected renal arterial stenosis on ultrasonography shows no renal arterial stenosis (score 1). 
conducted to validate the concept of time-SLIP SSFP technique as a screening tool for renal artery stenosis. Second, no patients with stenosis of distal segment of renal artery were included in our study, and it remains unclear how well non-contrast MRA performs for detection of distal renal arterial stenosis. Third, the time-SLIP SSFP will not always work. The image quality and vessel opacification of this technique considerably depends on in-flow phenomenon, and thus the slow vessel flow might cause the impaired diagnostic accuracy in patients with an aortic aneurysm. Fourth, the comparison between non-contrast MRA and ultrasonography was insufficient. Ultrasonography is recommended for initial screening of renal artery stenosis for impaired renal function ${ }^{3}$ Ultrasonography is the firstchoice examination for renal artery stenosis in our hospital. When the finding of ultrasonography was significant, MRA was not performed. In the present study, 6 patients underwent both ultrasonography and MRA because the patients with obesity and impaired breath-hold capability showed equivocal findings on the ultrasonography. The renal arteries of the 6 patients were normal on MRA (Fig 4). Comparison with other modalities such as ultrasonography and radionuclide renography should be conducted in the future study.

In conclusion, non-contrast MRA with time-SLIP SSFP technique is a non-invasive and effective method that allows evaluation of the renal artery stenosis. Furthermore, non-contrast MRA might potentially reduce the risk of NSF in patient with suspected renal artery stenosis.

\section{References}

1. Vasbinder GB, Nelemans PJ, Kessels AG, Kroon AA, de Leeuw $\mathrm{PW}$, van Engelshoven JM. Diagnostic tests for renal artery stenosis in patients suspected of having renovascular hypertension: A metaanalysis. Ann Intern Med 2001; 135: 401-411.
2. Galanski M, Prokop M, Chavan A, Schaefer C, Jandeleit K, Olbricht C. Accuracy of CT angiography in the diagnosis of renal artery stenosis. Rofo 1994; 161: 519-525 (in German).

3. Fleischmann D. Multiple detector-row CT angiography of the renal and mesenteric vessels. Eur J Radiol 2003; 45(Suppl): S79-S87.

4. Shiragami K, Fujii Z, Sakumura T, Shibuya M, Takahashi N, Yano $\mathrm{M}$, et al. Effect of a contrast agent on long-term renal function and the efficacy of prophylactic hemodiafiltration. Circ J 2008; 72: 427 433.

5. Kawashima S, Takano H, Iino Y, Takayama M, Takano T. Prophylactic hemodialysis does not prevent contrast-induced nephropathy after cardiac catheterization in patients with chronic renal insufficiency. Circ J 2006; 70: $553-558$.

6. Cowper SE, Robin HS, Steinberg SM, Su LD, Gupta S, LeBoit PE. Scleromyxoedema-like cutaneous diseases in renal-dialysis patients. Lancet 2000; 356: 1000-1001.

7. Marckmann P, Skov L, Rossen K, Dupont A, Damholt MB, Heaf JG, et al. Nephrogenic systemic fibrosis: Suspected causative role of gadodiamide used for contrast-enhanced magnetic resonance imaging. J Am Soc Nephrol 2006; 17: 2359-2362.

8. Mendoza FA, Artlett CM, Sandorfi N, Latinis K, Piera-Velazquez S, Jimenez SA. Description of 12 cases of nephrogenic fibrosing dermopathy and review of the literature. Semin Arthritis Rheum 2006; 35: $238-249$.

9. Grobner T. Gadolinium-a specific trigger for the development of nephrogenic fibrosing dermopathy and nephrogenic systemic fibrosis? Nephrol Dial Transplant 2006; 21: 1104-1108.

10. Maki JH, Wilson GJ, Eubank WB, Glickerman DJ, Millan JA, Hoogeveen RM. Navigator-gated MR angiography of the renal arteries: A potential screening tool for renal artery stenosis. Am J Roentgenol 2007; 188: W540-W546.

11. Hany TF, Leung DA, Pfammatter T, Debatin JF. Contrast-enhanced magnetic resonance angiography of the renal arteries: Original investigation. Invest Radiol 1998; 33: 653-659.

12. Utsunomiya D, Awai K, Tamura Y, Nishiharu T, Urata J, Sakamoto T, et al. 16-MDCT aortography with a low-dose contrast material protocol. Am J Roentgenol 2006; 186: 374-378.

13. Kawarada O, Yokoi Y, Morioka N, Takemoto K. Renal artery stenosis in cardio-and cerebrovascular disease: Renal duplex ultrasonography as an initial screening examination. Circ J 2007; 71: $1942-$ 1947. 